\title{
SOME NEW SUM PERFECT SQUARE GRAPHS
}

\author{
S.G. Sonchhatra ${ }^{1}$, G.V. Ghodasara ${ }^{2} \S$ \\ ${ }^{1}$ School of Science \\ R.K. University \\ Rajkot, INDIA \\ ${ }^{1}$ Government Engineering College \\ Rajkot, Gujarat, INDIA \\ ${ }^{2}$ H. H.B. Kotak Institute of Science \\ Rajkot, Gujarat, INDIA
}

\begin{abstract}
A $(p, q)$ graph $G=(V, E)$ is called sum perfect square if for a bijection $f$ : $V(G) \rightarrow\{0,1,2, \ldots, p-1\}$ there exists an injection $f^{*}: E(G) \rightarrow \mathbb{N}$ defined by $f^{*}(u v)=$ $(f(u))^{2}+(f(v))^{2}+2 f(u) \cdot f(v), \forall u v \in E(G)$. Here $f$ is called sum perfect square labeling of $G$. In this paper we derive several new sum perfect square graphs.
\end{abstract}

AMS Subject Classification: $05 \mathrm{C} 78$.

Key Words: Sum perfect square graph, half wheel graph

\section{Introduction}

We consider simple, finite, undirected graph $G=(p, q)$ (with p vertices and q edges). The vertex set and the edge set of $G$ are denoted by $V(G)$ and $E(G)$ respectively. For all other terminology and notations we follow Harary[1].

Sonchhatra and Ghodasara[4] initiated the study of sum perfect square graphs. Due to [4] it becomes possible to construct a graph, whose all edges can be labeled by different perfect square integers. In [4] the authors proved that $P_{n}, C_{n}, C_{n}$ with one chord, $C_{n}$ with twin chords, tree, $K_{1, n}, T_{m, n}$ are sum

Received: December 28, 2016

Revised: $\quad$ February 20, 2017

Published: $\quad$ March 28, 2017

(c) 2017 Academic Publications, Ltd. url: www.acadpubl.eu

$\S_{\text {Correspondence author }}$ 
perfect square graphs.

In this paper we prove that half wheel, corona, middle graph, total graph, $K_{1, n}+K_{1}, K_{2}+m K_{1}$ are sum perfect square graphs.

Definition 1.1. Let $G=(p, q)$ be a graph. A bijection $f: V(G) \rightarrow$ $\{0,1,2, \ldots, p-1\}$ is called sum perfect square labeling of $G$, if the induced function $f^{*}: E(G) \rightarrow \mathbb{N}$ defined by $f^{*}(u v)=(f(u))^{2}+(f(v))^{2}+2 f(u) \cdot f(v)$ is injective, $\forall u v \in E(G)$.

A graph which admits sum perfect square labeling is called sum perfect square graph.

Definition 1.2. The corona product $G \odot H$ of two graphs $G$ and $H$ is obtained by taking one copy of $G$ and $|V(G)|$ copies of $H$ and by joining each vertex of the $i^{t h}$ copy of $H$ to the $i^{t h}$ vertex of $G$ by an edge, $1 \leq i \leq|V(G)|$.

Definition 1.3. The middle graph of a graph $G$ denoted by $M(G)$ is the graph with vertex set $V(G) \cup E(G)$, where two vertices are adjacent if and only if either they are adjacent edges of $G$ or one is a vertex of $G$ and other is an edge incident with it.

Definition 1.4. The total graph of a graph $G$ denoted by $T(G)$ is the graph with vertex set is $V(G) \cup E(G)$ where two vertices are adjacent if and only if

(1) $x, y \in V(G)$ are adjacent.

(2) $x, y \in E(G)$ are adjacent.

(3) $x \in V(G), y \in E(G)$ and $y$ is incident to $x$.

Definition 1.5. Half wheel graph, denoted by $H W_{n}$ is constructed by the following steps.

Step 1: Consider a star $K_{1, n}$. Let $\left\{v_{1}, v_{2}, \ldots, v_{n}\right\}$ be the pendant vertices of $K_{1, n}$ and $v$ be the apex vertex of $K_{1, n}$.

Step 2: Add an edge between $v_{i}$ and $v_{i+1}, 1 \leq i \leq\left\lfloor\frac{n}{2}\right\rfloor$.

Note that $\left|V\left(H\left(W_{n}\right)\right)\right|=n+1,\left|E\left(H\left(W_{n}\right)\right)\right|=n+\left\lfloor\frac{n}{2}\right\rfloor$.

Definition 1.6. Let $G_{1}$ and $G_{2}$ be two graphs such that $V\left(G_{1}\right) \cap V\left(G_{2}\right)=$ $\phi$. The join of $G_{1}$ and $G_{2}$, denoted by $G_{1}+G_{2}$, is the graph with $V\left(G_{1}+G_{2}\right)=$ $V\left(G_{1}\right) \cup V\left(G_{2}\right)$ and $E\left(G_{1}+G_{2}\right)=E\left(G_{1}\right) \cup E\left(G_{2}\right) \cup J$, where $J=\left\{u v / u \in V\left(G_{1}\right)\right.$ and $\left.v \in V\left(G_{2}\right)\right\}$. 


\section{Main Results}

Observation 1: If $G=(V, E)$ is not sum perfect square graph, then its supergraph is also not sum perfect square graph, but the converse may not be true.
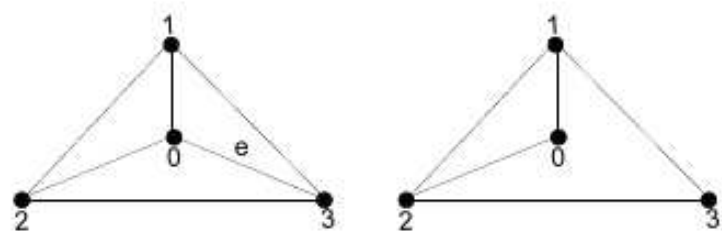

Figure 1: A non sum perfect square graph $K_{4}$ with sum perfect square subgraph $K_{4}-\{e\}$.

In [4] Sonchhatra and Ghodasara posed the following conjecture.

Conjecture 2.1. An odd simple graph $G$ with $\delta(G)=3$ is not sum perfect square.

Here we prove this conjecture by using the principle of mathematical induction on number of vertices of the graph.

Theorem 2.2. An odd simple graph $G$ with $\delta(G)=3$ is not sum perfect square.

Proof. For any graph $G=(V, E)$ with $|V(G)|=n$, since $d(v) \geq 3, \forall v \in G$, $n$ must be even and $n \geq 4$. We prove this conjecture by using principle of mathematical induction on number of vertices $n$ of graph $G$.

Step 1. For $n=4, G=K_{4}$ is not sum perfect square graph (See [4]).

Step 2. Suppose the result is true for $n=k, 4<k<n$.

Step 3. Let $G^{\prime}=\left(V^{\prime}, E^{\prime}\right)$ be the graph with $\left|V^{\prime}\right|=k+1 . H=G^{\prime}-\{v\}$ is the graph with $k$ vertices, where $v$ is any arbitrary vertex of $G^{\prime}$. By induction hypothesis, $H$ is not a sum perfect square graph. Since $G^{\prime}$ is a supergraph of $H$, it is not a sum perfect square graph (Observation 1).

Theorem 2.3. $C_{n} \odot K_{1}$ is sum perfect square graph, $n \geq 3$.

Proof. Let $V\left(C_{n} \odot K_{1}\right)=\left\{u_{i} ; 1 \leq i \leq n\right\} \cup\left\{v_{i} ; 1 \leq i \leq n\right\}$, where $u_{1}, u_{2}, \ldots, u_{n}$ are successive vertices of $C_{n}$ and $v_{1}, v_{2}, \ldots, v_{n}$ be the successive 
vertices corresponding to $n$ copies of $K_{1}$,

$$
\begin{aligned}
& E\left(C_{n} \odot K_{1}\right)=\left\{e_{i}^{(1)}=u_{i} u_{i+1} ; 1 \leq i \leq n-2\right\} \cup\left\{e_{n-1}^{(1)}=u_{n} u_{1}\right\} \\
& \cup\left\{e_{i}^{(2)}=u_{i} v_{i} ; 1 \leq i \leq n\right\} .
\end{aligned}
$$

We note that $\left|V\left(C_{n} \odot K_{1}\right)\right|=2 n$ and $\left|E\left(C_{n} \odot K_{1}\right)\right|=2 n$.

We define a bijection $f: V\left(C_{n} \odot K_{1}\right) \rightarrow\{0,1,2, \ldots, 2 n-1\}$ as

$$
\begin{aligned}
f\left(u_{1}\right) & =0 \\
f\left(u_{i}\right) & = \begin{cases}4 i-6 ; & 2 \leq i \leq\left\lfloor\frac{n}{2}\right\rfloor+1 . \\
4 n-4 i+4 ; & \left\lfloor\frac{n}{2}\right\rfloor+2 \leq i \leq n .\end{cases} \\
f\left(v_{i}\right) & =f\left(u_{i}\right)+1,1 \leq i \leq n .
\end{aligned}
$$

Let $f^{*}: E\left(C_{n} \odot K_{1}\right) \rightarrow \mathbb{N}$ be the induced edge labeling function defined by $f^{*}(u v)=(f(u))^{2}+(f(v))^{2}+2 f(u) \cdot f(v), \forall u v \in E\left(C_{n} \odot K_{1}\right)$.

Injectivity for edge labels: For $1 \leq i \leq\left\lfloor\frac{n}{2}\right\rfloor+1, f^{*}\left(e_{i}^{(1)}\right)$ is increasing in terms of $i \Rightarrow f^{*}\left(u_{i} u_{i+1}\right)<f^{*}\left(u_{i+1} u_{i+2}\right)$ and for $\left\lfloor\frac{n}{2}\right\rfloor+2 \leq i \leq n, f^{*}\left(e_{i}^{(1)}\right)$ is decreasing in terms of $i \Rightarrow f^{*}\left(u_{i} u_{i+1}\right)>f^{*}\left(u_{i+1} u_{i+2}\right)$. Similarly $f^{*}\left(e_{i}^{(2)}\right)$ is increasing for $1 \leq i \leq\left\lfloor\frac{n}{2}\right\rfloor+1$ and decreasing for $\left\lfloor\frac{n}{2}\right\rfloor+2 \leq i \leq n$.

Claim: $\left\{f^{*}\left(e_{i}^{(1)}\right), 1 \leq i \leq\left\lfloor\frac{n}{2}\right\rfloor+1\right\} \neq\left\{f^{*}\left(e_{i}^{(1)}\right),\left\lfloor\frac{n}{2}\right\rfloor+2 \leq i \leq n-1\right\} \neq$ $f^{*}\left(e_{n}^{(1)}\right) \neq\left\{f^{*}\left(e_{i}^{(2)}\right), 1 \leq i \leq\left\lfloor\frac{n}{2}\right\rfloor+1\right\} \neq\left\{f^{*}\left(e_{i}^{(2)}\right),\left\lfloor\frac{n}{2}\right\rfloor+2 \leq i \leq n\right\}$.

We have

$$
\begin{aligned}
f^{*}\left(e_{i}^{(1)}\right) & =\left\{\begin{array}{l}
(8 i-8)^{2} ; 2 \leq i \leq\left\lfloor\frac{n}{2}\right\rfloor . \\
(8 n-8 i+4)^{2} ;\left\lfloor\frac{n}{2}\right\rfloor+2 \leq i \leq n-1 .
\end{array}\right. \\
f^{*}\left(e_{\left\lfloor\frac{n}{2}\right\rfloor+1}^{(1)}\right) & =(4 n-6)^{2}, f^{*}\left(e_{1}^{(1)}\right)=2, f^{*}\left(e_{n}^{(1)}\right)=4 .
\end{aligned}
$$

Further

$$
f^{*}\left(e_{i}^{(2)}\right)=\left\{\begin{array}{l}
(8 i-11)^{2} ; 2 \leq i \leq\left\lfloor\frac{n}{2}\right\rfloor+1 \\
(8 n-8 i+9)^{2} ;\left\lfloor\frac{n}{2}\right\rfloor+2 \leq i \leq n
\end{array}\right.
$$

It is clear that

(1) $f^{*}\left(e_{1}^{(2)}\right)=1, f^{*}\left(e_{1}^{(1)}\right)=2$ and $f^{*}\left(e_{n}^{(1)}\right)=4$ are three smallest edge labels among all the edge labels in this graph.

(2) $\left\{f^{*}\left(e_{i}^{(1)}\right), 1 \leq i \leq n\right\}$ are even, $\left\{f^{*}\left(e_{i}^{(2)}\right), 1 \leq i \leq n\right\}$ are odd. 
(3) $f^{*}\left(e_{\left\lfloor\frac{n}{2}\right\rfloor+1}\right)$ is larger than the largest edge label of $\left\{f^{*}\left(e_{i}^{(1)}\right), 1 \leq i \leq\left\lfloor\frac{n}{2}\right\rfloor\right\}$ and smaller than the smallest edge label of $\left\{f^{*}\left(e_{i}^{(1)}\right),\left\lfloor\frac{n}{2}\right\rfloor+2 \leq i \leq n\right\}$.

Hence we only need to prove the following.

(1) $\left\{f^{*}\left(e_{1}^{(1)}\right) ; 2 \leq i \leq\left\lfloor\frac{n}{2}\right\rfloor\right\} \neq\left\{f^{*}\left(e_{i}^{(1)}\right),\left\lfloor\frac{n}{2}\right\rfloor+2 \leq i \leq n-1\right\}$.

(2) $\left\{f^{*}\left(e_{1}^{(2)}\right) ; 1 \leq i \leq\left\lfloor\frac{n}{2}\right\rfloor\right\} \neq\left\{f^{*}\left(e_{i}^{(2)}\right),\left\lfloor\frac{n}{2}\right\rfloor+2 \leq i \leq n\right\}$.

Assume if possible $\left\{f^{*}\left(e_{1}^{(1)}\right) ; 2 \leq i \leq\left\lfloor\frac{n}{2}\right\rfloor\right\}=\left\{f^{*}\left(e_{i}^{(1)}\right),\left\lfloor\frac{n}{2}\right\rfloor+2 \leq i \leq n-1\right\}$, for some $i$.

$\Longrightarrow 8 i-8=8 n-8 i+4$ or $8 i-8=8 i-8 n-4$.

$\Longrightarrow i=\frac{2 n+3}{4}$ or $n=\frac{1}{2}$, which contradicts with the choice of $i$ and $n$ as $i, n \in \mathbb{N}$. Assume if possible $\left\{f^{*}\left(e_{1}^{(2)}\right) ; 1 \leq i \leq\left\lfloor\frac{n}{2}\right\rfloor\right\} \neq\left\{f^{*}\left(e_{i}^{(2)}\right),\left\lfloor\frac{n}{2}\right\rfloor+2 \leq i \leq n\right\}$, for some $i$.

$\Longrightarrow 8 i-11=8 n-8 i+9$ or $8 i-11=8 i-8 n-9$.

$\Longrightarrow i=\frac{2 n+5}{4}$ or $n=\frac{1}{4}$, which contradicts with the choice of $i$ as $i \in \mathbb{N}$.

Hence $f^{*}: E\left(C_{n} \odot K_{1}\right) \rightarrow \mathbb{N}$ is injective. So $C_{n} \odot K_{1}$ is sum perfect square graph, $n \geq 3$.

The below illustration provides better idea about the above defined labeling pattern.

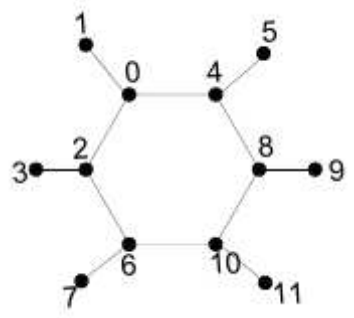

Figure 2: Sum perfect square labeling of $C_{6} \odot K_{1}$.

Theorem 2.4. $M\left(P_{n}\right)$ is sum perfect square graph, $\forall n \in \mathbb{N}$.

Proof. Let $V\left(M\left(P_{n}\right)\right)=\left\{v_{i} ; 1 \leq i \leq n\right\} \cup\left\{v_{i}^{\prime} ; 1 \leq i \leq n-1\right\}$, where $v_{i}^{\prime}$ is adjacent with $v_{i+1}^{\prime}, v_{i}$ and $v_{i+1}$, for $1 \leq i \leq n-1$. Here $E\left(M\left(P_{n}\right)\right)=\left\{e_{i}=\right.$ $\left.v_{i}^{\prime} v_{i+1}^{\prime} ; 1 \leq i \leq n-2\right\} \cup\left\{e_{i}^{(1)}=v_{i} v_{i}^{\prime} ; 1 \leq i \leq n-1\right\} \cup\left\{e_{i}^{(2)}=v_{i+1} v_{i}^{\prime} ; 1 \leq i \leq n-1\right\}$. We note that $\left|V\left(M\left(P_{n}\right)\right)\right|=2 n-1$ and $\left|E\left(M\left(P_{n}\right)\right)\right|=3 n-4$.

We define the bijection $f: V\left(M\left(P_{n}\right)\right) \rightarrow\{0,1,2, \ldots, 2 n-2\}$ as $f\left(v_{i}\right)=2 i-2,1 \leq i \leq n, f\left(v_{i}^{\prime}\right)=2 i-1,1 \leq i \leq n-1$. 
Let $f^{*}: E\left(M\left(P_{n}\right)\right) \rightarrow \mathbb{N}$ be the induced edge labeling function defined by $f^{*}(u v)=(f(u))^{2}+(f(v))^{2}+2 f(u) \cdot f(v), \forall u v \in E\left(M\left(P_{n}\right)\right)$.

Injectivity for edge labels: For $1 \leq i \leq n-2, f^{*}\left(e_{i}\right)$ is increasing in terms of $i \Rightarrow f^{*}\left(v_{i}^{\prime} v_{i+1}^{\prime}\right)<f^{*}\left(v_{i+1}^{\prime} v_{i+2}^{\prime}\right), 1 \leq i \leq n-3$. Similarly $f^{*}\left(e_{i}^{(1)}\right)$ and $f^{*}\left(e_{i}^{(2)}\right)$ are also increasing, $1 \leq i \leq n-1$.

Claim: $\left\{f^{*}\left(e_{i}\right) ; 1 \leq i \leq n-2\right\} \neq\left\{f^{*}\left(e_{i}^{(1)}\right) ; 1 \leq i \leq n-1\right\} \neq\left\{f^{*}\left(e_{i}^{(2)}\right) ; 1 \leq\right.$ $i \leq n-1\}$.

We have $f^{*}\left(e_{i}\right)=(4 i)^{2}, 1 \leq i \leq n-2$ and $f^{*}\left(e_{i}{ }^{(1)}\right)=(4 i-3)^{2}, f^{*}\left(e_{i}{ }^{(2)}\right)=$ $(4 i-1)^{2}, 1 \leq i \leq n-1$.

$f^{*}\left(e_{i}\right)$ are even, $1 \leq i \leq n-2$ and $f^{*}\left(e_{i}{ }^{(j)}\right)$ are odd, $1 \leq i \leq n-1, j=1,2$.

Assume if possible $f^{*}\left(e_{i}^{(1)}\right)=f^{*}\left(e_{i}^{(2)}\right)$, for some $i, 1 \leq i \leq n-1$.

$\Longrightarrow 4 i-3=4 i-1$ or $4 i-3=1-4 i$

$\Longrightarrow 3=1$ or $i=\frac{1}{2}$, which contradicts the choice of $i$, as $i \in \mathbb{N}$.

So $f^{*}: E\left(M\left(P_{n}\right)\right) \rightarrow \mathbb{N}$ is injective. Hence $M\left(P_{n}\right)$ is sum perfect square graph, $\forall n \in \mathbb{N}$.

The below illustration provides the better idea of the above defined labeling pattern.

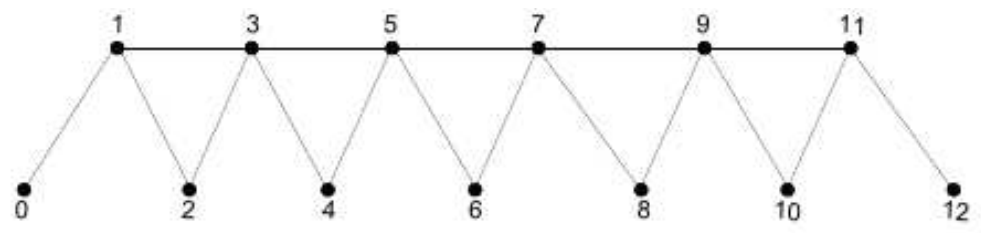

Figure 3 : Sum perfect square labeling of $M\left(P_{7}\right)$.

Theorem 2.5. $T\left(P_{n}\right)$ is sum perfect square graph, $\forall n \in \mathbb{N}$.

Proof. Let $V\left(T\left(P_{n}\right)\right)=\left\{v_{i} ; 1 \leq i \leq n\right\} \cup\left\{v_{i}^{\prime} ; 1 \leq i \leq n-1\right\}$, where $v_{i}^{\prime}$ is adjacent with $v_{i+1}^{\prime}, v_{i}$ and $v_{i+1}, 1 \leq i \leq n-1 . \quad E\left(T\left(P_{n}\right)\right)=\left\{e_{i}=\right.$ $\left.v_{i}^{\prime} v_{i+1}^{\prime} ; 1 \leq i \leq n-2\right\} \cup\left\{e_{i}^{(1)}=v_{i} v_{i}^{\prime} ; 1 \leq i \leq n-1\right\}$

$\cup\left\{e_{i}^{(2)}=v_{i+1} v_{i}^{\prime} ; 1 \leq i \leq n-1\right\} \cup\left\{e_{i}^{(3)}=v_{i} v_{i+1} ; 1 \leq i \leq n-1\right\} .\left|V\left(T\left(P_{n}\right)\right)\right|=$ $2 n-1$ and $\left|E\left(T\left(P_{n}\right)\right)\right|=4 n-5$.

We define a bijection $f: V\left(T\left(P_{n}\right)\right) \rightarrow\{0,1,2, \ldots, 2 n-2\}$ as $f\left(v_{i}\right)=2 i-2,1 \leq i \leq n, f\left(v_{i}^{\prime}\right)=2 i-1,1 \leq i \leq n-1$.

Let $f^{*}: E\left(T\left(P_{n}\right)\right) \rightarrow \mathbb{N}$ be the induced edge labeling function defined by $f^{*}(u v)=(f(u))^{2}+(f(v))^{2}+2 f(u) \cdot f(v), \forall u v \in E\left(T\left(P_{n}\right)\right)$. 
Injectivity for edge labels: For $1 \leq i \leq n-2, f^{*}\left(e_{i}\right)$ is increasing in terms of $i \Rightarrow f^{*}\left(v_{i}^{\prime} v_{i+1}^{\prime}\right)<f^{*}\left(v_{i+1}^{\prime} v_{i+2}^{\prime}\right), 1 \leq i \leq n-3$. Similarly $f^{*}\left(e_{i}^{(j)}\right)$ are also increasing, $1 \leq i \leq n-1,1 \leq j \leq 3$.

Claim: $\left\{f^{*}\left(e_{i}\right) ; 1 \leq i \leq n-2\right\} \neq\left\{f^{*}\left(e_{i}^{(1)}\right) ; 1 \leq i \leq n-1\right\} \neq\left\{f^{*}\left(e_{i}^{(2)}\right) ; 1 \leq\right.$ $i \leq n-1\} \neq\left\{f^{*}\left(e_{i}^{(3)}\right) ; 1 \leq i \leq n-1\right\}$.

$f^{*}\left(e_{i}\right)=(4 i)^{2}, 1 \leq i \leq n-2$. For $1 \leq i \leq n-1, f^{*}\left(e_{i}{ }^{(1)}\right)=(4 i-3)^{2}$, $f^{*}\left(e_{i}{ }^{(2)}\right)=(4 i-1)^{2}, f^{*}\left(e_{i}^{(3)}\right)=(4 i-2)^{2}$.

$\left\{f^{*}\left(e_{i}\right), 1 \leq i \leq n-2\right\}, f^{*}\left(e_{i}{ }^{(3)}\right)$ are even and $f^{*}\left(e_{i}{ }^{(j)}\right)$ are odd, for $1 \leq i \leq n-1$, $j=1,2,3$. It is enough to prove the following.

(1) $\left\{f^{*}\left(e_{i}\right), 1 \leq i \leq n-2\right\} \neq\left\{f^{*}\left(e_{i}^{(3)}\right), 1 \leq i \leq n-1\right\}$.

(2) $\left\{f^{*}\left(e_{i}^{(1)}\right), 1 \leq i \leq n-1\right\} \neq\left\{f^{*}\left(e_{i}^{(2)}\right), 1 \leq i \leq n-1\right\}$.

Assume if possible $\left\{f^{*}\left(e_{i}\right), 1 \leq i \leq n-2\right\}=\left\{f^{*}\left(e_{i}^{(3)}\right), 1 \leq i \leq n-1\right\}$, for some $i$.

$\Longrightarrow 4 i=4 i-2$ or $4 i=2-4 i$.

$\Longrightarrow 1=-2$ or $i=\frac{1}{4}$, which contradicts with the choice of $i$, as $i \in \mathbb{N}$.

Assume if possible $\left\{f^{*}\left(e_{i}^{(2)}\right), 1 \leq i \leq n-1\right\}=\left\{f^{*}\left(e_{i}^{(3)}\right), 1 \leq i \leq n-1\right\}$, for some $i$.

$\Longrightarrow 4 i-3=4 i-1$ or $4 i-3=1-4 i$.

$\Longrightarrow-3=-1$ or $i=\frac{1}{2}$, which contradicts with the choice of $i$, as $i \in \mathbb{N}$.

So $f^{*}: E\left(T\left(P_{n}\right)\right) \rightarrow \mathbb{N}$ is injective. Hence $T\left(P_{n}\right)$ is sum perfect square graph, $\forall n \in \mathbb{N}$.

The below illustration provides the better idea of the above defined labeling pattern.

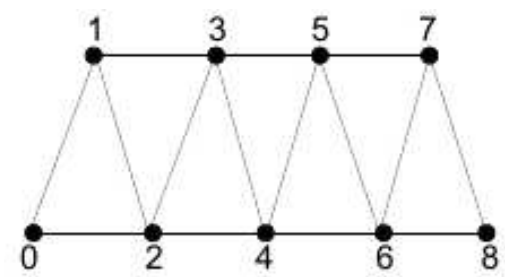

Figure 4 : Sum perfect square labeling of $T\left(P_{5}\right)$.

Theorem 2.6. $H W_{n}$ is sum perfect square graph, $\forall n \in \mathbb{N}$. 
Proof. Let $V\left(H W_{n}\right)=\{v\} \cup\left\{v_{i} ; 1 \leq i \leq n\right\}$ and $E\left(H W_{n}\right)=\left\{e_{i}=v v_{i} ; 1 \leq\right.$ $i \leq n\} \cup\left\{e_{i}^{(1)}=v_{i} v_{i+1} ; 1 \leq i \leq\left\lfloor\frac{n}{2}\right\rfloor\right\} .\left|V\left(H W_{n}\right)\right|=n+1$ and $\left|E\left(H W_{n}\right)\right|=$ $n+\left\lfloor\frac{n}{2}\right\rfloor$.

We define a bijection $f: V\left(H W_{n}\right) \rightarrow\{0,1,2, \ldots, n\}$ as $f(v)=n, f\left(v_{i}\right)=$ $i-1,1 \leq i \leq n$.

Let $f^{*}: E\left(H W_{n}\right) \rightarrow \mathbb{N}$ be the induced edge labeling function defined by $f^{*}(u v)=(f(u))^{2}+(f(v))^{2}+2 f(u) \cdot f(v), \forall u v \in E\left(H W_{n}\right)$.

Injectivity for edge labels: For $1 \leq i \leq n, f^{*}\left(e_{i}\right)$ is increasing in terms of $i \Rightarrow f^{*}\left(v v_{i}\right)<f^{*}\left(v v_{i+1}\right), 1 \leq i \leq n-1$. Similarly $f^{*}\left(e_{i}^{(1)}\right)$ is also increasing.

The largest edge label of $f^{*}\left(e_{i}^{(1)}\right)$ is smaller than the smallest edge label of $f^{*}\left(e_{i}\right)$, therefore $\left\{f^{*}\left(e_{i}\right) ; 1 \leq i \leq n\right\} \neq\left\{f^{*}\left(e_{i}^{(1)}\right) ; 1 \leq i \leq\left\lfloor\frac{n}{2}\right\rfloor\right\}$.

Hence the induced edge labeling $f^{*}: E\left(H\left(W_{n}\right)\right) \rightarrow \mathbb{N}$ is injective.

So $H W_{n}$ is sum perfect square graph, $\forall n \in \mathbb{N}$.

The below illustration gives the better understanding of above defined labeling pattern.
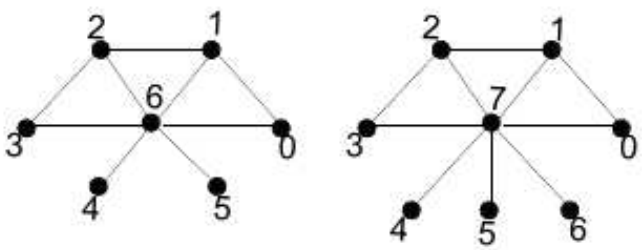

Figure 5: Sum perfect square labeling of $H\left(W_{6}\right)$ and $H\left(W_{7}\right)$.

Theorem 2.7. $K_{1, n}+K_{1}$ is sum perfect square graph, $\forall n \in \mathbb{N}$.

Proof. Let $V\left(K_{1, n}+K_{1}\right)=\{v\} \cup\left\{v_{i} ; 1 \leq i \leq n\right\} \cup\{w\}$, where $\left\{v_{1}, v_{2}, \ldots, v_{n}\right\}$ are the pendant vertices and $v$ is the apex vertex of $K_{1, n}$ and $w$ is the apex vertex corresponding to $K_{1}$. Here $E\left(K_{1, n}+K_{1}\right)=\left\{e_{i}^{(1)}=v v_{i} ; 1 \leq i \leq n\right\} \cup$ $\left\{e_{i}^{(2)}=w v_{i} ; 1 \leq i \leq n\right\} \cup\{e=v w\}$. Note that $\left|V\left(K_{1, n}+K_{1}\right)\right|=n+2$ and $\left|E\left(K_{1, n}+K_{1}\right)\right|=2 n+1$.

We define a bijection $f: V\left(K_{1, n}+K_{1}\right) \rightarrow\{0,1,2, \ldots, n+1\}$ as $f(v)=0, f\left(v_{i}\right)=i, 1 \leq i \leq n, f(w)=n+1$.

Let $f^{*}: E\left(K_{1, n}+K_{1}\right) \rightarrow \mathbb{N}$ be the induced edge labeling function defined by $f^{*}(u v)=(f(u))^{2}+(f(v))^{2}+2 f(u) \cdot f(v), \forall u v \in E\left(K_{1, n}+K_{1}\right)$.

Injectivity for edge labels: For $1 \leq i \leq n, f^{*}\left(e_{i}^{(1)}\right)$ is increasing in terms of $i \Rightarrow f^{*}\left(v v_{i}\right)<f^{*}\left(v v_{i+1}\right), 1 \leq i \leq n-1$. Similarly $f^{*}\left(e_{i}^{(1)}\right)$ is also increasing, 
for $1 \leq i \leq n$.

Claim: $\left\{f^{*}\left(e_{i}^{(1)}\right) ; 1 \leq i \leq n\right\} \neq\left\{f^{*}\left(e_{i}^{(2)}\right) ; 1 \leq i \leq n\right\} \neq f^{*}(e)$.

We have $f^{*}\left(e_{i}^{(1)}\right)=(i)^{2}, 1 \leq i \leq n, f^{*}\left(e_{i}{ }^{(2)}\right)=(n+i+1)^{2}, 1 \leq i \leq n$ and $f^{*}(e)=(n+1)^{2}$.

The largest edge label of $f^{*}\left(e_{i}^{(1)}\right)$ is smaller than the smallest edge label of $f^{*}\left(e_{i}{ }^{(2)}\right)$.

If $\left\{f^{*}\left(e_{i}^{(1)}\right), 1 \leq i \leq n\right\}=\left\{f^{*}(e)\right\}$ for some $i$, then $i=n+1$ or $i=-n-1$, which contradicts with the choice of $i$, as $i \in \mathbb{N}$.

Further the smallest edge label of $f^{*}\left(e_{i}{ }^{(2)}\right)$ is larger than $f^{*}(e)$. Therefore $\left\{f^{*}\left(e_{i}^{(2)}\right) ; 1 \leq i \leq n\right\} \neq f^{*}(e)$.

So $f^{*}: E\left(K_{1, n}+K_{1}\right) \rightarrow \mathbb{N}$ is injective.

Hence $K_{1, n}+K_{1}$ is sum perfect square graph.

The below illustration provides the better idea of the above defined labeling pattern.

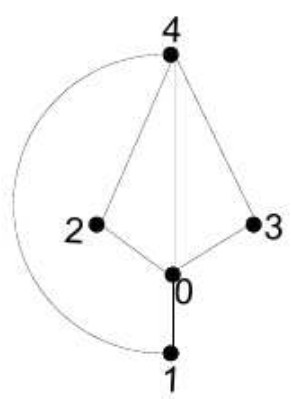

Figure 6 : Sum perfect square labeling of $K_{1,3}+K_{1}$.

Theorem 2.8. $K_{2}+m K_{1}$ is sum perfect square graph, $\forall m \in \mathbb{N}$.

Proof. Let $V\left(K_{2}+m K_{1}\right)=\left\{u_{1}, u_{2}\right\} \cup\left\{v_{i} ; 1 \leq i \leq m\right\}$, where $\left\{u_{1}, u_{2}\right\}$ be the vetex set of $K_{2}$. $E\left(K_{2}+m K_{1}\right)=\left\{e=u_{1} u_{2}\right\} \cup\left\{e_{i}^{(1)}=u_{1} v_{i} ; 1 \leq i \leq m\right\} \cup$ $\left\{e_{i}^{(2)}=u_{2} v_{i} ; 1 \leq i \leq m\right\}$. Here $\left|V\left(K_{2}+m K_{1}\right)\right|=m+2$ and $\left|E\left(K_{2}+m K_{1}\right)\right|=$ $2 m+1$.

We define the bijection $f: V\left(K_{2}+m K_{1}\right) \rightarrow\{0,1,2, \ldots, m+1\}$ as $f\left(u_{1}\right)=0, f\left(u_{2}\right)=m+1$ and $f\left(v_{i}\right)=i, 1 \leq i \leq m$.

Let $f^{*}: E\left(K_{2}+m K_{1}\right) \rightarrow \mathbb{N}$ be the induced edge labeling function defined by $f^{*}(u v)=(f(u))^{2}+(f(v))^{2}+2 f(u) \cdot f(v), \forall u v \in E\left(K_{2}+m K_{1}\right)$.

Injectivity for edge labels: For $1 \leq i \leq m$, since $f\left(v_{i}\right)$ is increasing in terms of $i \Rightarrow f^{*}\left(u_{j} v_{i}\right)<f^{*}\left(u_{j} v_{i+1}\right), j=1,2,1 \leq i \leq m-1$. 
Claim: $f^{*}(e) \neq\left\{f^{*}\left(e_{i}^{(1)}\right) ; 1 \leq i \leq m\right\} \neq\left\{f^{*}\left(e_{i}^{(2)}\right) ; 1 \leq i \leq m\right\}$.

We have $f^{*}(e)=(m+1)^{2}, f^{*}\left(e_{i}^{(1)}\right)=(i)^{2}, f^{*}\left(e_{i}^{(2)}\right)=(m+i+1)^{2}, 1 \leq i \leq$ $m$.

The largest edge label of $f^{*}\left(e_{i}{ }^{(1)}\right)$ is smaller than the smallest edge label of $f^{*}\left(e_{i}{ }^{(2)}\right)$. Also $f^{*}(e)$ is larger than the highest edge label of $f^{*}\left(e_{i}{ }^{(1)}\right)$ and smaller than the smallest edge label of $f^{*}\left(e_{i}{ }^{(2)}\right)$. Hence the claim is proved. So the induced edge labeling $f^{*}: E\left(K_{2}+m K_{1}\right) \rightarrow \mathbb{N}$ is injective. So $K_{2}+m K_{1}$ is sum perfect square graph, $\forall m \in \mathbb{N}$.

The below illustration provides the better idea of the above defined labeling pattern.

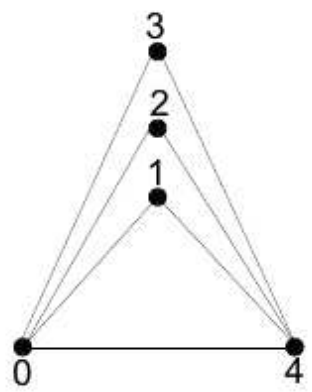

Figure 7 : Sum perfect square labeling of $K_{2}+3 K_{1}$.

\section{Conclusion}

In this paper a conjecture related to sum perfect square graph have been proved, a new graph called half wheel have been presented and various sum perfect square graphs are found. 


\section{References}

[1] F. Harary, Graph Theory, Addision-wesley, Reading, MA, (1969).

[2] J.A. Gallian, A dynemic survey of graph labeling, The Electronics Journal of Combinatorics, 18 (2015) 1-262.

[3] J. Shiama, Square sum labeling for some middle and total graphs, International Journal of Computer Applications, 37 (2012) 6-8, doi: 10.5120/4594-6548.

[4] S.G. Sonchhatra, G.V. Ghodasara, Sum perfect square labeling of graphs, International Journal of Scientific and Innovative Mathematical Research, 4 (2016) 64-70. 
